\title{
Prognostic Factors of Vitreous Hemorrhage Secondary to Exudative Age-Related Macular Degeneration
}

\section{$\operatorname{AUTHOR}(S)$ :}

Hasegawa, Tomoko; Otani, Atsushi; Sasahara, Manabu; Gotoh, Norimoto; Ooto, Sotaro; Tamura, Hiroshi; Yamashiro, Kenji; Tsujikawa, Akitaka; Yoshimura, Nagahisa

\section{CITATION:}

Hasegawa, Tomoko ... [et al]. Prognostic Factors of Vitreous Hemorrhage Secondary to Exudative Age-Related Macular Degeneration. American Journal of Ophthalmology 2010, 149(2): 322-329.e1

\section{ISSUE DATE:}

2010-02-01

URL:

http://hdl.handle.net/2433/92977

\section{RIGHT:}

(C) 2010 Elsevier Inc. All rights reserved.; この論文は出版社版でありませ ん。引用の際には出版社版をご確認ご利用ください。; This is not the published version. Please cite only the published version. 


\section{Elsevier Editorial System(tm) for American Journal of Ophthalmology Manuscript Draft}

Manuscript Number: AJO-09-10R3

Title: Prognostic Factors of Vitreous Hemorrhage Secondary to Exudative Age-related Macular Degeneration

Article Type: Original Article

Keywords: vitreous hemorrhage; age-related macular degeneration; pars plana vitrectomy; prognostic factors

Corresponding Author: Atsushi Otani, M.D. Ph.D.

Corresponding Author's Institution: Kyoto University

First Author: Hasegawa Tomoko, MD

Order of Authors: Hasegawa Tomoko, MD; Atsushi Otani, MD; Manabu Sasahara, MD; Norimoto Gotoh, MD; Sotaro Ooto, MD; Hiroshi Tamura, MD; Kenji Yamashiro, MD; Akitaka Tsujikawa, MD; Nagahisa Yoshimura, MD 


\section{ABSTRACT}

PURPOSE: Vitreous hemorrhage $(\mathrm{VH})$ is a rare but serious complication of the eyes with exudative age-related macular degeneration (AMD). This retrospective study was designed to evaluate various clinical factors that may affect the visual prognosis of patients with VH secondary to exudative AMD.

DESIGN: Retrospective case study.

METHODS: We intensively documented 31 cases of VH secondary to exudative AMD and retrospectively analyzed best-corrected visual acuity (BCVA). All eyes underwent standard pars plana vitrectomy (PPV) for treating $\mathrm{VH}$. Three subgroups were created according to the clinical course and treatment history before the occurrence of $\mathrm{VH}$ : (1) Gas group (7 eyes), Pneumatic displacement with $\mathrm{SF}_{6}$ gas performed to treat massive submacular hemorrhage; (2) PDT group (9 eyes), Photodynamic therapy performed to treat exudative AMD; (3) Untreated group (15 eyes), No treatment performed.

RESULTS: As a whole, BCVA before the occurrence of $\mathrm{VH}$ was $1.05 \pm 0.59$ (LogMAR). After the occurrence of $\mathrm{VH}, \mathrm{BCVA}$ before PPV dropped to $2.61 \pm 0.82$. After the operation, final BCVA significantly improved to $1.25 \pm 0.73\left(P<10^{-8}\right)$. In a subgroup analysis, no statistically significant difference was seen among the three subgroups at any time point. We found that the eyes whose fellow eye had exudative AMD showed significantly poor final BCVA compared to the unilateral cases $(0.92 \pm 0.57$ and $1.49 \pm 0.72, P=0.02)$.

CONCLUSIONS: PPV can improve visual acuity in the eyes with $\mathrm{VH}$ secondary to AMD, although effectiveness is limited. Medical practitioners should be cautious of the visual prognosis, especially in the cases whose fellow eye has exudative AMD.

(Total: 244 words) 


\section{INTRODUCTION}

Choroidal neovascularization (CNV) secondary to exudative age-related macular degeneration (AMD) has a poor visual prognosis due to fundus manifestations that include pigment epithelial detachment, retinal detachment, sub-retinal/macular hemorrhage, hard exudates, macular edema, and subretinal fibrosis. Vitreous hemorrhage $(\mathrm{VH})$ is a rare but serious complication of exudative AMD. ${ }^{1-3} \mathrm{VH}$ itself contributes to vision loss in AMD and is considered a sign of AMD progression. Although unstable neovasculature may be related to $\mathrm{VH}$, the precise mechanisms that lead to ocular VH with exudative AMD remain unclear. In some cases, VHs may be spontaneously absorbed over time; however, pars plana vitrectomy (PPV) is often required to remove the hemorrhage. PPV surgery provides a relatively good visual prognosis. $^{4,5}$

In ocular VH with exudative AMD, submacular hemorrhage often precedes the occurrence of spontaneous $\mathrm{VH}^{2}{ }^{2} \mathrm{VH}$ can occur in cases of massive submacular hemorrhage following treatments such as pneumatic displacement. ${ }^{6,7}$ Photodynamic therapy (PDT) for the treatment of exudative AMD can also cause $\mathrm{VH}^{8,9}$ However, there is little information about how the clinical course of the disease affects visual prognosis in eyes with $\mathrm{VH}$ due to exudative AMD.

To clarify the factors that affect the prognosis of $\mathrm{VH}$ secondary to exudative AMD, we documented the clinical courses of patients with exudative AMD that underwent PPV to remove VH. Clinical factors likely to influence visual prognosis were investigated. We additionally evaluated the efficacy of pars plana vitrectomy (PPV) for treating $\mathrm{VH}$ due to exudative AMD. 


\section{METHODS}

For this interventional case study, we retrospectively reviewed the medical records of 979 consecutive patients with exudative AMD who were treated at the Macula Service, Department of Ophthalmology, Kyoto University Hospital, from January 2004 to September 2008. Inclusion criteria were as follows: (1) the occurrence of VH during the follow-up period in the clinic, (2) a diagnosis of exudative AMD during followup, (3) treatment with PPV for $\mathrm{VH}$, and (4) a postoperative follow-up period greater than three months. Patients were divided into 3 subgroups depending on their clinical course before the occurrence of $\mathrm{VH}$ : (1) gas group: pneumatic displacement with $\mathrm{SF}_{6}$ gas was used to treat massive submacular hemorrhage before the occurrence of VH; (2) PDT group: PDT was used to treat exudative AMD before the occurrence of $\mathrm{VH}$; and (3) untreated group: no treatment was performed before the occurrence of $\mathrm{VH}$. For the purposes of this study, patients with polypoidal choroidal vasculopathy (PCV) and retinal angiomatous proliferation (RAP) were considered to have exudative AMD and were included in the study. We use the term AMD to mean exudative AMD that could not be diagnosed as PCV or RAP. Patients with other types of CNV, i.e. pathologic myopia, angioid streaks, RAP, idiopathic CNV, presumed ocular histoplasmosis syndrome, and other secondary CNV were excluded from this study. Patients with other retinal/macular abnormalities, i.e., retinal macroaneurysm, diabetic retinopathy, retinal vein occlusion, or uveitis, were also excluded from the study.

A PCV diagnosis was based on an indocyanine green (ICG) angiogram that showed polypoidal structures along the border of the branching vascular network. A polypoidal lesion can appear as a single polyp or as a cluster of polyps. In most PCV cases, reddish-orange subretinal nodules were seen under ophthalmoscopic examination that corresponded to the polypoidal lesions seen by ICG angiography. All patients had comprehensive ophthalmologic examinations, including best-corrected visual acuity (BCVA) measurements, binocular ophthalmoscopy, slit-lamp biomicroscopy with a contact lens, color fundus photography, fluorescein angiography, ICG angiography, and optical coherence tomography (OCT). Fluorescein angiography and ICG angiography were performed simultaneously using a confocal laser scanning system (HRA-2; Heidelberg Engineering, Dossenheim, Germany). Four types of OCT instruments were used (Stratus OCT, Carl Zeiss, Dublin, CA; OCT-ophthalmoscope, Nidek, Gamagori, Japan; 3D OCT-1000, Topcon, Tokyo, Japan; RTVue, Optovue Corp., San Francisco, CA). All patients were assessed carefully at baseline and then 
every 3 months. In addition, patients were advised to visit the clinic if they felt their vision was worsening.

To treat VH, standard three-port PPV was performed on all patients in the study using a 20-gauge or 23-gauge system. Six experienced surgeons in our macular clinic performed all surgeries. Phacoemulsification and intraocular lens implantation was combined in all phakic eyes because of the expected progressive nuclear sclerosis. Fluid-air exchange and sulfur hexafluoride $\left(\mathrm{SF}_{6}\right)$ gas tamponade was performed in some cases.

To treat massive submacular hemorrhage, pneumatic displacement was performed using $0.5 \mathrm{ml}$ of $100 \% \mathrm{SF}_{6}$ gas injected into the vitreous cavity with a 30 gauge needle. Patients were instructed to maintain a prone position at least 4 days. PDT with verteporfin to treat exudative AMD was performed according to standard procedures. A 689-nm laser system (Carl Zeiss, Dublin, CA) was used, and 50-J/mm² energy was delivered with an 83-second exposure time.

For statistical analysis, counting fingers (CF), hand motion (HM) and light perception (LP) were considered to indicate decimal visual acuity of $0.005,0.001$ and 0.0005 , respectively. The BCVA was determined using the Landolt ring test and converted into the logarithm of the minimum angle of resolution (logMAR). All values are reported as the mean \pm standard deviation. Continuous variables between two groups were compared using the Student's t-test (two-tailed). Continuous variables between three groups were compared by one-way analysis of variance. Multivariate linear regression analysis was performed to correlate final visual acuity with other clinical parameters. All analyses were performed using a commercial software program (SPSS v.13.0; SPSS, Chicago, IL). A difference was considered statistically significant when the $P$ value was less than 0.05 . 


\section{RESULTS}

This study included 31 patients, each with one eye with $\mathrm{VH}$ due to exudative AMD (3.2\% of the 979 age-related exudative AMD patients) (Table 1). The patients ranged in age from 55 to 86 years (mean \pm standard deviation, $73.8 \pm 7.7$ years). The mean postoperative follow-up period was $19.9 \pm 14.2$ months (range, 5-52 months). Of the 31 eyes, 22 (71\%) were diagnosed as PCV by angiographic analysis; the other 9 were diagnosed as AMD. Patient characteristics are summarized in Table 1. Submacular hemorrhage and hemorrhagic pigment epithelial detachment was detected prior to $\mathrm{VH}$ in 14 and 15 eyes, respectively. Seven patients presented with $\mathrm{VH}$ at their initial visit to the clinic. The CNV lesion was located parafoveally in patients $2,12,13$, $14,15,17,18$, and 21 ( $n=8)$ (Table 1).

Vitreous hemorrhage was observed after pneumatic displacement of submacular hemorrhage using intravitreal injection of $\mathrm{SF}_{6}$ gas in 7 eyes (the gas group); the mean period from gas injection to the occurrence of $\mathrm{VH}$ was $51 \pm 102$ days (range, 3-300 days). Before VH, PDT was performed in 9 eyes (the PDT group). In the PDT group, the mean period from the last PDT to the occurrence of VH was $14 \pm 12$ months (range, 0.5-31 months). In the other 15 eyes (the untreated group), no treatment was performed before $\mathrm{VH}$ was detected. PPV was performed in all cases to remove the VH and was performed in conjunction with phacoemulsification in 25 eyes (81\%).

The mean BCVA (at the last documentation for each eye) before $\mathrm{VH}$ in the 25 patients who were seen in the clinic before the $\mathrm{VH}$ occurred was $1.05 \pm 0.59$ LogMAR units. After the occurrence of $\mathrm{VH}$, the mean BCVA decreased to $2.61 \pm 0.82$ and then showed significant improvement 3 months after PPV surgery $\left(P<10^{-8}\right)$; improvements in BCVA were maintained during the follow-up period (Figure 1, top). The mean BCVA 6 months after surgery was $1.23 \pm 0.78$ and was $1.12 \pm 0.8212$ months after surgery $(P<$ $10^{-8}$ ). Although the improvement in BCVA after surgery was more than 0.3 LogMAR units in 26 eyes (84\%), the final BCVA was not satisfactory in most cases. Only 8 eyes $(26 \%)$ had a final BCVA $\geq 1.0$ LogMAR units. When analyzing the five cases with relatively good visual prognosis (less than LogMAR 0.6, cases 4, 7, 12, 18, and 21), we found that all cases were PCV and that four patients $(80 \%)$ were unilaterally affected. In addition, all five cases had polypoidal lesions, pigment epithelial detachment, or submacular hemorrhage that was para- or extrafoveal. Three of the 5 eyes (60\%) showed branching vascular networks under the fovea (cases 7, 18, and 21).

In the subgroup analysis, BCVA before the occurrence of $\mathrm{VH}$ was $0.82 \pm 0.30$ in the gas group, $0.97 \pm 0.26$ in the PDT group, and $1.08 \pm 0.65$ in the untreated group. 
After the occurrence of $\mathrm{VH}, \mathrm{BCVA}$ before PPV was $2.46 \pm 0.87$ in the gas group, $2.54 \pm 0.92$ in the PDT group, and $2.71 \pm 0.79$ in the untreated group. The final BCVA was $0.90 \pm 0.56$ in the gas group, $1.35 \pm 0.72$ in the PDT group, and $1.34 \pm 0.79$ in the untreated group. There seemed to be a trend for the gas group to have a better visual prognosis compared to other groups; however, no statistically significant difference was seen in the three subgroups at any time point (Figure 1, middle). In our series, 7 of the 9 PDT cases that developed VH had only one PDT session. In the other 2 cases (cases 9 and 16), the patient received multiple PDT sessions before $\mathrm{VH}$. In most of the PDT cases, the lesion was stabilized after PDT, and VH occurred with the recurrence of AMD. In 2 cases (cases 9 and 11 in Table 1), PDT did not stabilize the lesion; subsequently, $\mathrm{VH}$ occurred.

Interestingly, the percentage of bilaterally affected exudative AMD patients was high for the VH cases (16 eyes, 52\%) compared to all the exudative AMD cases in our clinic (21\%). Therefore, we compared unilaterally $(n=15)$ and bilaterally $(n=16)$ affected patients. BCVA before the occurrence of $\mathrm{VH}$ was $1.01 \pm 0.72$ in the unilateral group and $1.07 \pm 0.48$ in the bilateral group, while BCVA before PPV was $2.69 \pm 0.62$ and $2.55 \pm 0.96$, respectively. There were significant differences in BCVA between the two groups at 3 months $(0.95 \pm 0.54$ vs $1.56 \pm 0.82 ; P=0.02), 6$ months $(0.87 \pm 0.56$ vs $1.55 \pm 0.83 ; P=0.01), 12$ months $(0.71 \pm 0.58$ vs $1.53 \pm 0.84 ; P=0.02)$, and at the final end point $(P=0.02)$ (Figure 1, bottom).

Using multivariate regression analysis, we also examined other factors that might have influenced the 6-month BCVA (Table 2). Explanatory variables were age, sex, hypertension, diabetes mellitus, anticoagulant medication, history of smoking, angiographic subtype, submacular hemorrhage before $\mathrm{VH}$, phacoemulsification and intraocular lens implantation combined vitrectomy, logMAR before $\mathrm{VH}$, logMAR before PPV, and logMAR of the fellow eye. None of these factors showed statistical significance.

Avastin injection was used in cases 1, 6, 28, and 30. In all of these cases, the injection was performed once in the period after $\mathrm{VH}$ and surgery. 


\section{DISCUSSION}

In our series of 31 eyes with $\mathrm{VH}$ secondary to CNV, PCV occurred in a high percentage of cases (71\%). PCV is a subtype of exudative AMD, and the first published case of PCV was reported as posterior uveal bleeding syndrome. ${ }^{10}$ Subsequent to that report, there have been numerous studies of PCV. As detected by ICG angiography, polypoidal vasculature rather than massive bleeding is now considered a common and distinctive angiographic feature of the disease. Bleeding is still an important characteristic of PCV, and the rate of hemorrhagic complications, including hemorrhagic pigment epithelial detachment in PCV, is higher than in other types of AMD. ${ }^{11,12}$

$\mathrm{VH}$ is a hemorrhagic complication of exudative AMD that can cause severe vision loss. Although the incidence of $\mathrm{VH}$ in exudative AMD is not high (3.2\% of 979 cases in our case series), the incidence of PCV among the $\mathrm{VH}$ cases was higher $(71 \%)$ than the incidence of PCV among all exudative AMD cases (47\% in our clinic). PCV should thus be considered when patients with exudative AMD present with $\mathrm{VH}$.

Anticoagulant medications can contribute to the incidence of hemorrhagic complications in exudative AMD, for example in submacular hemorrhage or $\mathrm{VH}^{1,13}$ In the current study, only $16 \%$ of the 31 patients were on anticoagulants, and in our previous report on hemorrhagic complications in PCV cases after PDT, anticoagulants did not affect the incidence of complications. ${ }^{9}$ Larger case series are needed to fully explore this issue. During the observation period in this study, medications were not related to changes in BCVA.

In this study, the clinical courses leading to $\mathrm{VH}$ did not affect the significant differences we observed in BCVA (3-month mean BCVA: gas group $0.94 \pm 0.59$, PDT group $1.36 \pm 0.65$, untreated group $1.36 \pm 0.86$; 6-month mean BCVA: gas group $0.79 \pm$ 0.55, PDT group 1.35 \pm 0.78 , untreated group $1.38 \pm 0.85$; 12-month mean BCVA: gas group $0.70 \pm 0.57$, PDT group $1.28 \pm 0.90$, untreated group $1.19 \pm 0.86)$. Although the difference was not statistically significant $(P=)$, eyes affected by $\mathrm{VH}$ in the six months after PDT tended to have a lower final BCVA than other eyes in the PDT group. There is no evidence that PDT itself triggers $\mathrm{VH}$, but we previously found that $6.6 \%$ of PVC resulted in $\mathrm{VH}$ following $\mathrm{PDT}{ }^{9}$ It is possible that $\mathrm{VH}$ after PDT may result from incomplete tissue and vascular repair, indicating a poor prognosis. Further studies are required to clarify this issue; in the meantime, clinicians should focus on cases in which VH occurs immediately after PDT.

In our series of 979 patients, there were 15 patients who were treated with pneumatic displacement for submacular hemorrhage. Of these, 7 developed $\mathrm{VH}$ and 
were included in this study. The final mean visual acuity for these 7 cases was $0.90 \pm$ $0.56 \log M A R$, whereas that of the other 8 cases who did not develop VH was $0.88 \pm$ 0.6 . Because there was no significant difference between the two groups, it seems that development of $\mathrm{VH}$ after gas injection may have little effect on the prognosis of submacular hemorrhage. As shown in Figure 1, there was a tendency for the gas group to have better visual acuity compared to the untreated group. Gas injection should be considered for submacular hemorrhage, even if the treatment is a risk factor for $\mathrm{VH}$. We calculated the risks associated with several factors that might affect the incidence of $\mathrm{VH}$ after pneumatic displacement. However, intra-ocular lens implantation, hypertension $(\mathrm{HT})$, diabetes mellitus (DM), history of smoking, PDT, and anticoagulant medication were not correlated with the incidence of $\mathrm{VH}$.

We found that cases that were bilaterally affected by exudative AMD had very poor visual prognosis after $\mathrm{VH}$ compared to unilateral cases. We previously reported a bilaterally affected case with a different a biological response to PDT treatment that had a worse visual prognosis compared to a unilaterally affected case.$^{14}$ In addition, there is a loss of control of pathological vessels and tissues by circulating hematopoietic stem/progenitor cells in bilaterally affected cases. ${ }^{15}$ We noted a high percentage of bilaterally affected cases (52\%) in our VH case series (this study). In general, the incidence of bilateral exudative AMD in Japanese patients is $10-20 \%{ }^{16,17}$ This extremely high rate of bilateral exudative AMD in VH cases suggests a relationship between susceptibility to $\mathrm{VH}$ and bilateral affection. In genomic studies, patients with high-risk homozygous ARMS2 polymorphisms tend to have bilateral AMD, ${ }^{18,19}$ and this high-risk homozygous polymorphism is frequently seen in patients with $\mathrm{VH}^{20}$ These data suggest a common genetic background that that increases susceptibility to $\mathrm{VH}$ and bilateral exudative AMD. These reports, along with the data in the current study, raise the possibility that different biological responses or pathological causes may underlie the differences between bilateral and unilateral exudative AMD cases. Further investigations should clarify the relationship between susceptibility to $\mathrm{VH}$ and bilateral exudative AMD.

In conclusion, the current study searched for prognostic factors of $\mathrm{VH}$ secondary to exudative AMD. We found that (1) there is a high susceptibility to PCV in cases with VH secondary to exudative AMD, (2) PPV can restore visual acuity to a pre-VH level, although relatively good visual prognosis can only be obtained in limited cases, and (3) visual prognosis in bilaterally affected patients is poor. Although we included more patients in this study than were included in previous studies, an even larger case series 
is necessary for clarify the prognosis of $\mathrm{VH}$ in exudative AMD cases. Further efforts should focus on the biological responses and background of bilateral exudative AMD cases. 


\section{ACKNOWLEDGEMENT}

a) Funding/Support: None.

b) Financial disclosure: None for all authors.

c) Author contributions: Conception and design (T.H., A.O., M.S., N.Y.), analysis and interpretation (T.H., A.O., M.S., N.G., S.O., H.T., K.Y., A.T., N.Y.), manuscript preparation (T.H., A.O., M.S.), manuscript revision (N.G., S.O., H.T., K.Y., A.T., N.Y.), final approval of the article (T.H., A.O., M.S., N.G., S.O., H.T., K.Y., A.T., N.Y.), data collection (T.H., A.O., M.S.), provision of patients (A.O., S.O., H.T., K.Y., A.T., N.Y.), statistical analysis (T.H., A.O., M.S.), literature search (T.H., A.O., M.S.).

d) Conformity statement: This study was approved by the IRB of Kyoto University Hospital. This study adhered to the principles set forth in the Declaration of Helsinki.

e) Other acknowledgements: None. 


\section{REFERENCES}

1. el Baba F, Jarrett WH, 2nd, Harbin TS, Jr., et al. Massive hemorrhage complicating age-related macular degeneration. Clinicopathologic correlation and role of anticoagulants. Ophthalmology 1986;93:1581-1592.

2. Googe JM, Hirose T, Apple DJ, Melgen S. Vitreous hemorrhage secondary to age-related macular degeneration. Surv Ophthalmol 1987;32:123-130.

3. Azzolini C, Menchini U, Pece A, Camesasca F, Giuliani V. Age-related macular degeneration and vitreous hemorrhage. Eur J Ophthalmol 1991;1:142-147.

4. de Juan E, Jr., Machemer R. Vitreous surgery for hemorrhagic and fibrous complications of age-related macular degeneration. Am J Ophthalmol 1988;105:25-29. 5. Gomi F, Emi K, Motokura M, Oshima Y, Yamanishi S. [Long-term results after simple vitrectomy for age-related macular degeneration with vitreous hemorrhage]. Nippon Ganka Gakkai Zasshi 1998;102:436-441.

6. Hassan AS, Johnson MW, Schneiderman TE, et al. Management of submacular hemorrhage with intravitreous tissue plasminogen activator injection and pneumatic displacement. Ophthalmology 1999;106:1900-1906; discussion 1906-1907.

7. Daneshvar H, Kertes PJ, Leonard BC, Peyman GA. Management of submacular hemorrhage with intravitreal sulfur hexafluoride: a pilot study. Can J Ophthalmol 1999;34:385-388.

8. Ojima Y, Tsujikawa A, Otani A, Hirami Y, Aikawa H, Yoshimura N. Recurrent bleeding after photodynamic therapy in polypoidal choroidal vasculopathy. Am J Ophthalmol 2006;141:958-960.

9. Hirami Y, Tsujikawa A, Otani A, et al. Hemorrhagic complications after photodynamic therapy for polypoidal choroidal vasculopathy. Retina 2007;27:335-341. 10. Kleiner RC, Brucker AJ, Johnston RL. The posterior uveal bleeding syndrome. Retina 1990;10:9-17.

11. Yannuzzi LA, Sorenson J, Spaide RF, Lipson B. Idiopathic polypoidal choroidal vasculopathy (IPCV). Retina 1990;10:1-8.

12. Uyama M, Matsubara T, Fukushima I, et al. Idiopathic polypoidal choroidal vasculopathy in Japanese patients. Arch Ophthalmol 1999;117:1035-1042.

13. Tilanus MA, Vaandrager W, Cuypers MH, Verbeek AM, Hoyng CB. Relationship between anticoagulant medication and massive intraocular hemorrhage in age-related macular degeneration. Graefes Arch Clin Exp Ophthalmol 2000;238:482-485.

14. Kurashige $Y$, Otani $A$, Sasahara $M$, et al. Two-year results of photodynamic therapy for polypoidal choroidal vasculopathy. Am J Ophthalmol 2008;146:513-519. 


\begin{abstract}
15. Yodoi Y, Sasahara M, Kameda T, Yoshimura N, Otani A. Circulating
\end{abstract} hematopoietic stem cells in patients with neovascular age-related macular degeneration. Invest Ophthalmol Vis Sci 2007;48:5464-5472.

16. Uyama M, Takahashi K, Ida N, et al. The second eye of Japanese patients with unilateral exudative age related macular degeneration. Br J Ophthalmol 2000;84:10181023.

17. Maruko I, lida T, Saito M, Nagayama D, Saito K. Clinical characteristics of exudative age-related macular degeneration in Japanese patients. Am J Ophthalmol 2007;144:15-22.

18. Chen $\mathrm{H}$, Yang Z, Gibbs D, et al. Association of HTRA1 polymorphism and bilaterality in advanced age-related macular degeneration. Vision Res 2008;48:690-694. 19. Gotoh N, Yamada R, Nakanishi $\mathrm{H}$, et al. Correlation between $\mathrm{CFH}$ Y $402 \mathrm{H}$ and HTRA1 rs11200638 genotype to typical exudative age-related macular degeneration and polypoidal choroidal vasculopathy phenotype in the Japanese population. Clin Experiment Ophthalmol 2008;36:437-442.

20. Sakurada $\mathrm{Y}$, Kubota T, Mabuchi F, Imasawa M, Tanabe N, lijima H. Association of LOC387715 A69S with vitreous hemorrhage in polypoidal choroidal vasculopathy. Am J Ophthalmol 2008;145:1058-1062. 


\section{FIGURE LEGENDS}

Figure 1. Changes in visual acuity before and after pars plana vitrectomy (PPV) for vitreous hemorrhage (VH) secondary to age-related macular degeneration (AMD). (Top) LogMAR units before VH (pre-VH), after VH (pre-PPV), and final visual acuity after PPV (final). *: $P<10^{-6}$, compared to pre-PPV. (Middle) Comparison of LogMAR changes among the three subgroups. Gas = patients treated by intravitreal injection of $\mathrm{SF}_{6}$ gas for displacing submacular hemorrhage before the occurrence of VH. PDT = patients treated by photodynamic therapy for exudative AMD before the occurrence of $\mathrm{VH}$. Untreated = patients without any treatment before the occurrence of $\mathrm{VH}$. (Bottom) Comparison of LogMAR changes between patients with unilateral and bilateral exudative AMD. *: $P<0.05$.

Figure 2. A case of vitreous hemorrhage $(\mathrm{VH})$ secondary to exudative age-related macular degeneration resulting in relatively good visual prognosis (case 7). (Top left) The right eye is normal, with decimal visual acuity of 1.2. (Top right) The left eye showed massive submacular hemorrhage and hemorrhagic pigment epithelial detachment (PED) before the occurrence of $\mathrm{VH}$. (Bottom left) Indocyanine green angiogram showed a branching vascular network and polypoidal structure at the border of the network. Fluorescence is blocked at the PED lesions. (Bottom right) Pars plana vitrectomy (PPV) was performed after VH developed. Macular hemorrhage was absorbed and the decimal visual acuity of the left eye improved to 1.0 nine months after PPV.

Figure 3. A case of vitreous hemorrhage $(\mathrm{VH})$ secondary to exudative age-related macular degeneration (AMD) resulting in poor visual prognosis (case 15). (Top left) $A$ fundus photograph of the right eye before the occurrence of $\mathrm{VH}$. Large hemorrhagic pigment epithelial detachment (PED) can be seen beyond the vascular arcade. Orange subretinal lesions were observed at the border of the PED. The decimal visual acuity was 0.2. (Top right) The left eye showed fibrovascular scar formation secondary to old exudative AMD. (Bottom left) Indocyanine green angiogram of the right eye showed a large vascular network at the macula and aneurismal structures at the border of the network. (Bottom right) After the occurrence of $\mathrm{VH}$, the patient underwent PPV. A huge choroidal neovascular membrane was seen at the macula. Ten months after PPV, the decimal visual acuity was 0.02 . 
TABLE 1. Clinical Profile of 31 patients with Vitreous Hemorrhage Secondary to Age-related Macular Degeneration Treated by Pars Plana Vitrectomy.

\begin{tabular}{|c|c|c|c|c|c|c|c|c|c|c|c|c|c|c|c|}
\hline case & $\begin{array}{l}\text { Age } \\
\text { (y) }\end{array}$ & Sex & HT & DM & $\begin{array}{c}\text { Anticoagulant } \\
\text { Medication }\end{array}$ & $\begin{array}{l}\text { History of } \\
\text { Smoking }\end{array}$ & $\begin{array}{l}\text { Laterality } \\
\text { of CNV }\end{array}$ & Diagnosis & $\begin{array}{c}\text { Submacular } \\
\text { Hemorrhage } \\
\text { before VH }\end{array}$ & $\begin{array}{c}\text { Treatment } \\
\text { before VH } \\
\text { (day) }\end{array}$ & $\begin{array}{c}\text { Contents of } \\
\text { Surgery }\end{array}$ & $\begin{array}{c}\text { VA } \\
\text { before } \\
\text { VH }\end{array}$ & $\begin{array}{c}\text { VA } \\
\text { before } \\
\text { PPV }\end{array}$ & $\begin{array}{c}\text { VA } \\
\text { Final }\end{array}$ & $\begin{array}{l}\text { Postoperative } \\
\text { Follow-up } \\
\text { Periods (m) }\end{array}$ \\
\hline 1 & 71 & $\mathbf{M}$ & No & No & Yes & No & Unilateral & PCV & Yes & Gas (7) & PPV+PEA+IOL & 0.3 & N/A & 0.1 & 29 \\
\hline 2 & 71 & M & No & No & No & Yes & Bilateral & PCV & Yes & Gas (10) & PPV+PEA+IOL & 0.2 & 0.1 & 0.1 & 6 \\
\hline 3 & 78 & M & No & No & No & Yes & Unilateral & PCV & Yes & Gas (19) & PPV+PEA+IOL & 0.1 & HM & 0.04 & 5 \\
\hline 4 & 70 & M & No & No & No & Yes & Bilateral & AMD & Yes & Gas (7) & PPV+PEA+IOL & 0.08 & CF & 0.3 & 24 \\
\hline 5 & 68 & M & No & No & No & No & Unilateral & AMD & Yes & Gas (300) & PPV+PEA+IOL & 0.06 & HM & 0.02 & 52 \\
\hline 6 & 68 & M & Yes & Yes & Yes & No & Bilateral & AMD & Yes & Gas (10) & PPV+PEA+IOL & 0.15 & HM & 0.2 & 48 \\
\hline 7 & 71 & M & Yes & No & No & No & Unilateral & PCV & Yes & Gas (6) & PPV+PEA+IOL & 0.4 & N/A & 1.0 & 13 \\
\hline 8 & 81 & M & Yes & No & No & No & Bilateral & AMD & No & PDT (360) & PPV & 0.04 & CF & 0.07 & 15 \\
\hline 9 & 73 & $\mathbf{M}$ & Yes & No & No & Yes & Bilateral & PCV & No & PDT (45) & PPV+PEA+IOL & 0.1 & LP & HM & 13 \\
\hline 10 & 78 & M & Yes & No & No & Yes & Bilateral & PCV & No & PDT (180) & PPV & 0.15 & 0.07 & 0.06 & 37 \\
\hline 11 & 81 & $\mathbf{M}$ & No & No & No & Yes & Bilateral & PCV & Yes & PDT (15) & PPV & 0.07 & 0.07 & 0.05 & 8 \\
\hline 12 & 70 & $\mathbf{M}$ & No & No & No & No & Unilateral & PCV & No & PDT (930) & PPV+PEA+IOL & 0.3 & 0.01 & 0.5 & 12 \\
\hline 13 & 76 & M & No & No & No & Yes & Unilateral & PCV & No & PDT (810) & PPV+PEA+IOL & 0.1 & HM & 0.07 & 11 \\
\hline 14 & 85 & M & Yes & No & Yes & No & Bilateral & PCV & No & PDT (870) & PPV+PEA+IOL & 0.07 & LP & 0.08 & 8 \\
\hline 15 & 68 & M & No & No & No & Yes & Bilateral & PCV & No & PDT (420) & PPV+PEA+IOL & 0.2 & LP & 0.02 & 10 \\
\hline 16 & 55 & $\mathbf{F}$ & No & No & No & No & Unilateral & PCV & No & PDT (60) & PPV+PEA+IOL & 0.1 & LP & 0.06 & 23 \\
\hline 17 & 80 & $\mathbf{M}$ & Yes & No & No & Yes & Unilateral & PCV & Unknown & Untreated & PPV+PEA+IOL & N/A & HM & 0.2 & 25 \\
\hline 18 & 77 & $\mathbf{F}$ & Yes & No & No & No & Unilateral & PCV & Yes & Untreated & PPV+PEA+IOL & 0.3 & HM & 1.0 & 29 \\
\hline 19 & 58 & M & Yes & No & No & Yes & Unilateral & PCV & Yes & Untreated & $P P V+P E A+I O L$ & 0.06 & HM & 0.02 & 20 \\
\hline 20 & 78 & $\mathbf{M}$ & No & No & No & Yes & Bilateral & PCV & Yes & Untreated & PPV+PEA+IOL & 0.04 & LP & 0.03 & 11 \\
\hline 21 & 65 & M & Yes & No & No & Yes & Unilateral & PCV & Unknown & Untreated & PPV+PEA+IOL & N/A & CF & 0.4 & 12 \\
\hline 22 & 58 & M & No & No & No & Yes & Unilateral & PCV & Unknown & Untreated & PPV+PEA+IOL & N/A & 0.09 & 0.2 & 8 \\
\hline 23 & 80 & $\mathbf{M}$ & Yes & Yes & Yes & No & Bilateral & PCV & Unknown & Untreated & PPV & N/A & LP & HM & 6 \\
\hline 24 & 75 & $\mathbf{F}$ & Yes & No & No & No & Unilateral & PCV & Unknown & Untreated & PPV+PEA+IOL & N/A & CF & 0.08 & 8 \\
\hline
\end{tabular}




\begin{tabular}{|c|c|c|c|c|c|c|c|c|c|c|c|c|c|c|c|}
\hline 25 & 82 & M & No & No & Yes & Yes & Bilateral & PCV & No & Untreated & PPV & 0.02 & LP & CF & 17 \\
\hline 26 & 74 & M & Yes & No & No & Yes & Bilateral & AMD & Yes & Untreated & PPV+PEA+IOL & 0.08 & HM & 0.09 & 52 \\
\hline 27 & 73 & $\mathbf{F}$ & No & No & No & Yes & Unilateral & AMD & Yes & Untreated & PPV & 0.2 & HM & 0.1 & 7 \\
\hline 28 & 77 & $\mathbf{M}$ & Yes & No & No & Yes & Bilateral & AMD & Yes & Untreated & PPV+PEA+IOL & 0.01 & LP & 0.01 & 34 \\
\hline 29 & 86 & $\mathbf{F}$ & Yes & No & No & Yes & Unilateral & AMD & Unknown & Untreated & $P P V+P E A+1 O L$ & HM & HM & 0.02 & 8 \\
\hline 30 & 79 & M & Yes & Yes & No & No & Bilateral & PCV & Unknown & Untreated & PPV+PEA+IOL & N/A & 0.15 & 0.1 & 43 \\
\hline 31 & 81 & M & No & No & No & No & Bilateral & AMD & No & Untreated & PPV+PEA+IOL & 1.0 & HM & 0.01 & 22 \\
\hline
\end{tabular}

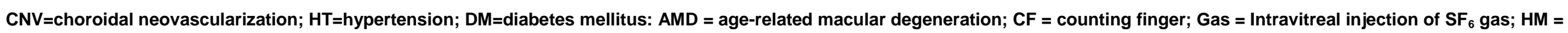

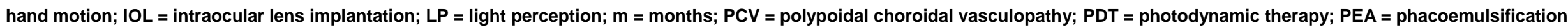

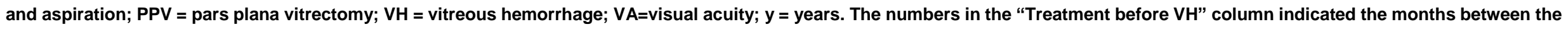
treatment and VH 
TABLE 2. Multivariate analysis of the factors which might have influenced the BCVA

\begin{tabular}{ccc}
\hline Independent Variable & $\beta$ coefficient & $P$ value \\
\hline Age & -0.20 & 0.47 \\
Sex & -0.45 & 0.11 \\
Hypertension & 0.04 & 0.90 \\
Diabetes mellitus & -0.29 & 0.34 \\
Medication of anticoagulant & 0.19 & 0.53 \\
History of smoking & 0.25 & 0.42 \\
Angiographic subtype of PCV & -0.01 & 0.98 \\
Submacular hemorrhage before VH & -0.11 & 0.72 \\
PEA+IOL combined & -0.20 & 0.52 \\
LogMAR of pre VH & -0.06 & 0.86 \\
LogMAR of pre PPV & 0.43 & 0.14 \\
LogMAR of fellow eye & -0.07 & 0.81 \\
\hline
\end{tabular}

$\mathrm{IOL}$ = intraocular lens implantation; $\mathrm{PCV}=$ polypoidal choroidal vasculopathy; $\mathrm{PEA}=$ phacoemulsification and aspiration; $P P V=$ pars plana vitrectomy; VH = vitreous hemorrhage. 

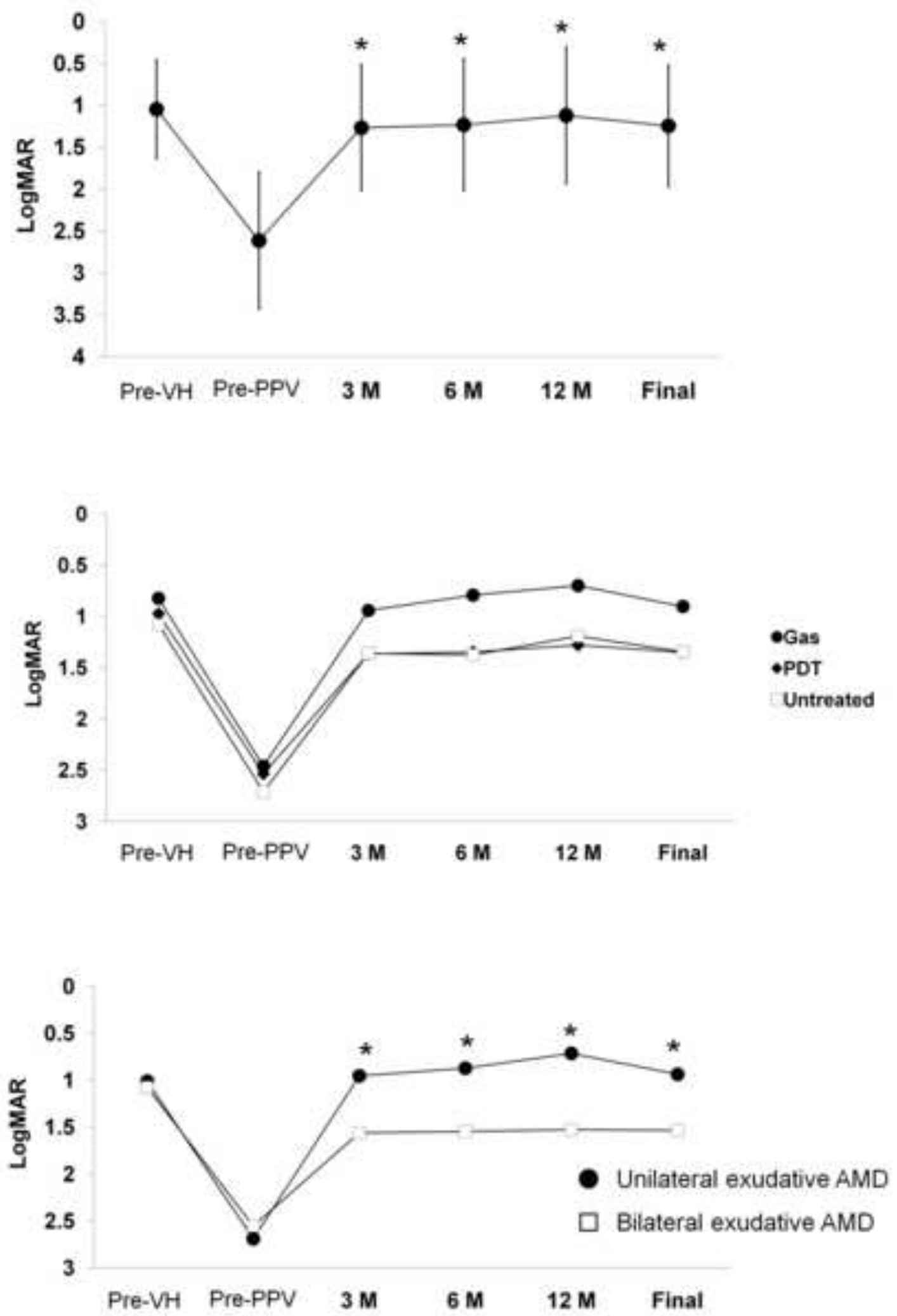


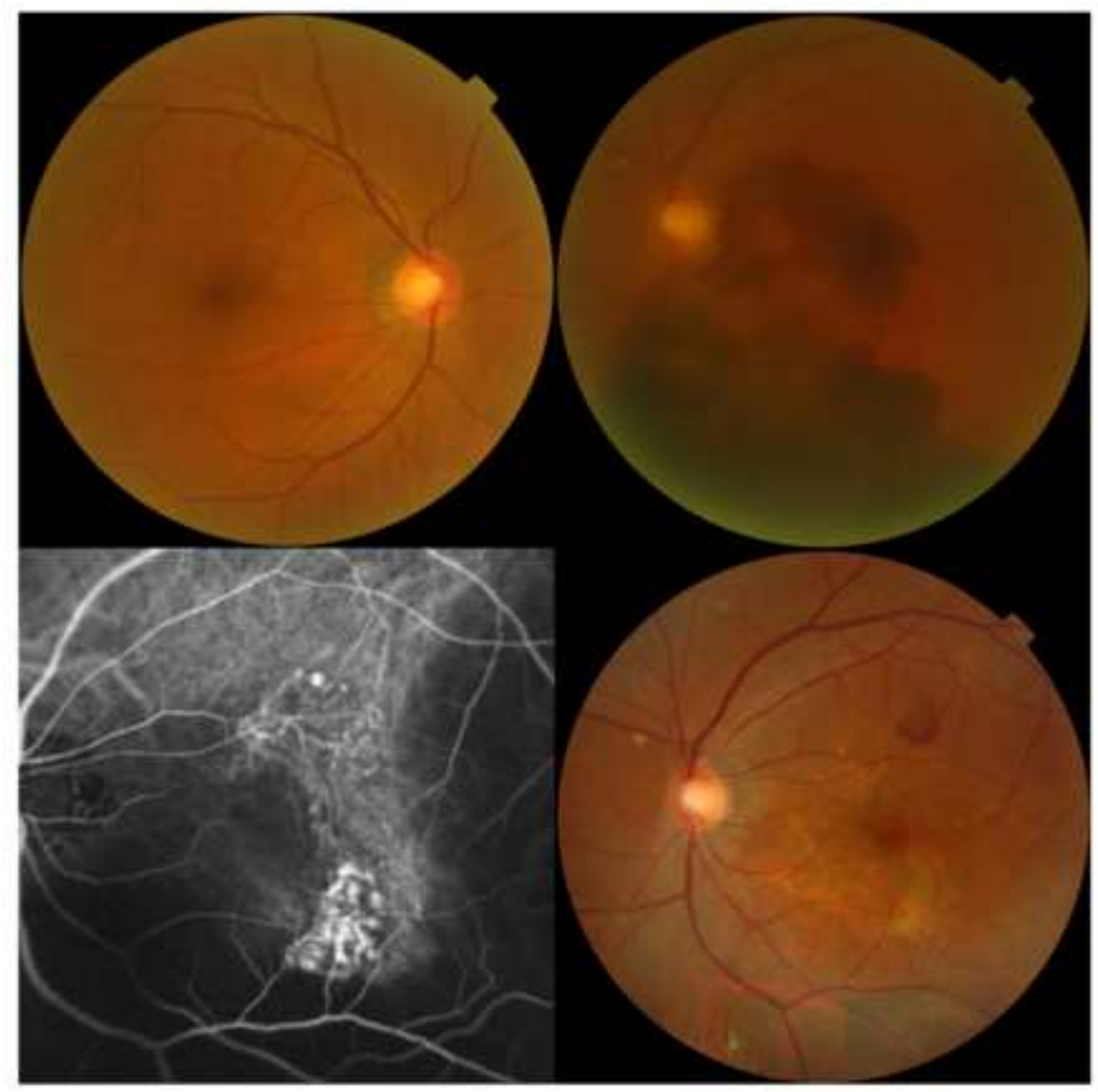


F: 京都大学

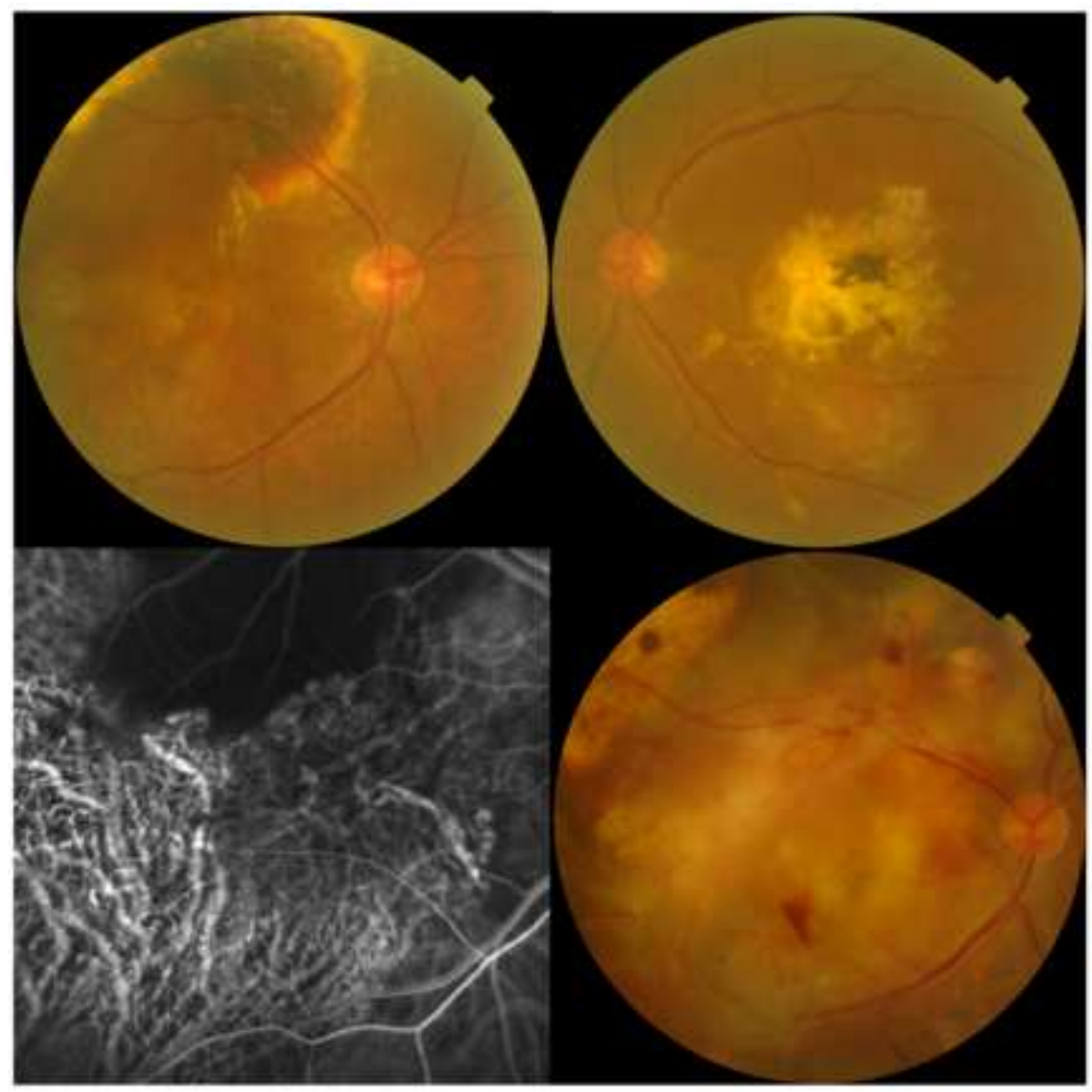

